\title{
Low myo-inositol indicating astrocytic damage in a case series of NMO
}

\begin{tabular}{|c|c|}
\hline Journal: & Annals of Neurology \\
\hline Manuscript ID: & ANA-11-0062.R3 \\
\hline Wiley - Manuscript type: & Brief Communication \\
\hline Date Submitted by the Author: & $\mathrm{n} / \mathrm{a}$ \\
\hline Complete List of Authors: & $\begin{array}{l}\text { Ciccarelli, Olga; UCL Institute of Neurology, Department of Brain Repair } \\
\text { and Rehabilitation } \\
\text { Thomas, David; UCL Institute of Neurology, Department of Brain Repair } \\
\text { and Rehabilitation } \\
\text { De Vita, Enrico; UCL Institute of Neurology, Lysholm Department of } \\
\text { Neuroradiology } \\
\text { Wheeler-Kingshott, Claudia; UCL Institute of Neurology, Department of } \\
\text { Neuroinflammation } \\
\text { Kachramanoglou, Carolina; UCL Institute of Neurology, Department of } \\
\text { Brain Repair and Rehabilitation } \\
\text { Kapoor, Raj; UCL Institute of Neurology, of Neuroinflammation } \\
\text { Leary, Siobhan; National Hospital for Neurology and Neurosurgery, } \\
\text { Matthews, Lucy; John Radcliffe Hospital, of Clinical Neurology } \\
\text { Palace, Jacqueline; John Radcliffe Hospital, of Clinical Neurology } \\
\text { Chard, Declan; University College London (UCL) Institute of Neurology, } \\
\text { NMR Research Unit; UCL Institute of Neurology, of Neuroinflammation } \\
\text { Miller, David; UCL Institute of Neurology, ; UCL Institute of Neurology, of } \\
\text { Neuroinflammation } \\
\text { Toosy, Ahmed; Institute of Neurology, Department of Brain Repair and } \\
\text { Rehabilitation } \\
\text { Thompson, Alan; UCL Institute of Neurology, Department of Brain Repair } \\
\text { and Rehabilitation }\end{array}$ \\
\hline Keywords: & NMO, MS, spinal cord \\
\hline Domain: & Clinical and/or Desktop Research \\
\hline
\end{tabular}

\section{SCHOLARONE ${ }^{\text {'T }}$}

Manuscripts 


\section{Low myo-inositol indicating astrocytic damage in a case series}

\section{of NMO}

Running head: In-vivo astrocytic damage in NMO

O Ciccarelli ${ }^{1} \stackrel{2}{2}, \mathrm{PhD}, \mathrm{DL}$ Thomas $^{1}, \mathrm{PhD}, \mathrm{E}$ De $\mathrm{Vita}^{1, \underline{\underline{3}} 2}, \mathrm{PhD}, \mathrm{CAM}$ Wheeler-

Kingshott $^{43}$, PhD, C Kachramanoglou ${ }^{1}$, MRCP, R Kapoor ${ }^{2,53}$, FRCP, S Leary ${ }^{54}$, MD, L Matthews ${ }^{\underline{6}}$, PhD, J. Palace ${ }^{65}$, FRCP, D Chard ${ }^{2.43}$, PhD, DH Miller $r^{2.43}$, FRCP, AT Toosy ${ }^{1}, \mathrm{PhD}$, AJ Thompson ${ }^{1,2}$, FRCP.

${ }^{1}$ Department of Brain Repair and Rehabilitation, NMR Research Unit, UCL Institute of Neurology, London, UK;

${ }^{2}$ National Institute for Health Research (NIHR) University College London Hospitals (UCLH) Biomedical Research Centre (BRC).

${ }^{3}$ Lysholm Department of Neuroradiology, National Hospital for Neurology and Neurosurgery, London UK;

${ }^{43}$ Department of Neuroinflammation, NMR Unit, UCL Institute of Neurology, London, UK;

${ }^{54}$ National Hospital for Neurology and Neurosurgery, Queen Square, London, UK; ${ }^{65}$ Department of Clinical Neurology, John Radcliffe Hospital, Oxford, UK.

Corresponding author: Dr Olga Ciccarelli; Department of Brain Repair and Rehabilitation, Institute of Neurology, University College London, Queen Square, London WC1N 3BG, UK; Telephone: 08451555000 (ext. 84469); e-mail: o.ciccarelli@ucl.ac.uk

Key words: NMO, MS, spinal cord, MR spectroscopy.

Number of characters in the title and running head: 69 and 32. Number of words ( in the abstract, and the body of the manuscript: 9998 and 15192440493 (including acknowledgments). Number of color figures: 1. 


\begin{abstract}
Astrocytic necrosis is a prominent pathological feature of Neuromyelitis Optica (NMO) lesions and is clinically relevant. We report five NMO-related cases, all with longitudinally extensive lesions in the upper cervical cord, who underwent cervical cord ${ }^{1} \mathrm{H}-\mathrm{MR}$ spectroscopy. Lower myo-Inositol/Creatine values, suggesting astrocytic damage, were consistently found within the NMO lesions when compared with eleven healthy controls and ten patients with multiple sclerosis (MS), who showed at least one demyelinating lesion at the same cord level. Therefore, the in vivo quantification of myo-Inositol may distinguish NMO from MS. This is an important step towards developing imaging markers for clinical trials in NMO.
\end{abstract}


Neuromyelitis Optica (NMO) comprises recurrent myelitis, with spinal cord lesions which extend over three or more vertebral segments, and optic neuritis. ${ }^{1}$ NMO is a disease primarily of astrocytes, due to antibodies against aquaporin-4 (AQP4-Abs), expressed at the astrocytic perivascular endfeet ${ }^{2}$. The interaction between AQP4-Abs and aquaporin-4 induces several molecular outcomes including oedema, inflammation, demyelination and astrocytic necrosis ${ }^{3}$. When these pathological processes affect the cervical cord, they can be studied in-vivo using advanced spinal cord imaging techniques ${ }^{4}$.

We performed spinal cord ${ }^{1} \mathrm{H}-\mathrm{MR}$ spectroscopy (MRS) in two patients with NMO and three patients with NMO spectrum disorder, each showing a longitudinally extensive transverse myelitis (LETM) in the upper cervical cord (i.e., involving either completely or partially the cord between C1 and C3). The main clinical and radiological characteristics of these patients are described in Table 1.

We also scanned ten patients with relapsing-remitting multiple sclerosis (MS) showing at least one demyelinating lesion in the spine volving the same cord levelbetween C1 and C3 (i.e., the same cord level as NMO cases), and eleven healthy subjects. The levels of myo-Inositol and $\mathrm{N}$-acetyl-aspartate normalised to Creatine, obtained with MRS, were compared between groups (see supplemental material for details on protocol and analysis).

\section{Case 1}


A 38-year old man had Guillain-Barre syndrome at the age of 4 with residual, although minimal, limb weakness. In 2009 he presented with sudden onset rightsided weakness and numbness which spread to the left. Spinal cord MRI showed a longitudinally-extensive lesion from the cranio-cervical junction to C5 with associated swelling and foci of enhancement. Treatment with IV corticosteroids resulted in an incomplete recovery. He was diagnosed with NMO spectrum disorder was diagnosed. He commenced Prednisolone and Azathioprine. His repeat spinal cord MRI showed improvement in the lesion, which extended from C1 to C5, two small foci of enhancement at $C 2$ and $C 4 / 5$, and no swelling. Five months later, he suffered another transverse myelitis. His MRI showed a more conspicuous lesion associated with swelling. He received IV steroids and an interval MRI (one month later) showed resolution of the swelling with the lesion extending from $\mathrm{C} 2$ to $\mathrm{C} 5$ and signal change in the cranio-cervical junction. In 2010 he developed post-corticosteroid left central serous retinopathy.

Seventeen months after his last transverse myelitisTM he underwent spinal cord MRS (Figure 1, Table 2). Examination revealed; at this time, hHis examination showed normal visual acuity, mild to moderate quadriparesis akness in all limbs, especially in the right arm, absent reflexes, and reduced right-sided sensation to light touch-on the right side.

\section{Case 2}


A 44-year old woman presented in 2001 with severe right optic neuritis, followed by transverse myelitis $\underline{T M}$, from which she made a partial recovery. In 2003 she developed transverse myelitis TM, with severe quadriparesis, sensory and sphincter disturbance, and was diagnosed with MS. With rehabilitation, she was able to walk with a frame. The following year her transverse myelitisTM recurred. Spinal cord MRI showed a longitudinally-extensive lesion extending from the foramen magnum to $\mathrm{C6}$. Her diagnosis was revised to $\mathrm{NMO}$ and she was treated with IV corticosteroids, Mitoxantrone and Azathioprine. In 2010 she presented

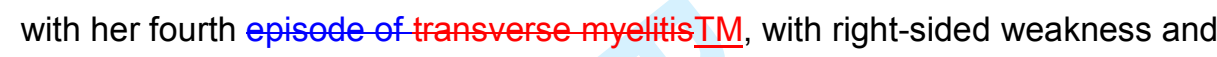
urinary dysfunction. She commenced on Prednisolone and continued on Azathioprine.

One year after her last transverse myelitis TM, she underwent spinal cord MRS (Table 2) $;$ her eExamination revealed a spastic monoplegia of her right leg and moderate weakness in her left leg and right arm,-a sensory level at C5, urinary urgency, and visual loss to light perception in her right eye.

\section{Case 3}

A 50-year old woman presented in May 2011 with sudden interscapular pain, and chest tightness. She then developed a paraparesis with left leg numbness. Spinal cord MRI scan showed hyperintense lesions at $\mathrm{C} 2$ and $\mathrm{C} 3 / \mathrm{C} 4$, and a longitudinal extensive lesionLETM from C6 to the conus, with which was continuous and associated with-oedema. All these cord lesions showed patchy enhancement. 
Despite IV steroids she progressed to a complete paraplegia, severe pain and reduced sitting ability. She was then treated with plasmapheresis, and commenced oral Prednisolone with Azathioprine. With rehabilitation she made a partial recovery. In July 2011 her spinal cord MRI showed a significant reduction in signal change throughout the cordin the cervical and thoracic cord, which became patchy and less confluent.

Four months after the onset of LETM she underwent spinal cord MRS (Table 2). Eher neurological examination showed complete monoplegia of her left leg, moderate to severe weakness in her right leg, sensory level at T4 and urinary dysfunction. She then underwent spinal cord MRS (Table 2).

\section{Case 4}

A 34-year old man presented in 2007 with hiccups; his vision deteriorated and he was admitted to ITU with respiratory failure due to a brainstem lesion. $\mathrm{He}$ developed severe leg weakness due to cervical LETM. He made a partial improvement following plasmapheresis. In 2008 he presented with severe optic neuritis, which did not improve. In 2009 he re-presented with $\underline{\text { TMa spinal cord }}$ relapse, making a good recovery after plasmapheresis. At that time, his spinal cord MRI scan showed a longitudinally-extensive lesion in the entire cervical cord, plus another lesion from T9 to T11. NMO was diagnosed; and he commenced Prednisolone and Azathioprine. In 2010 he had further bilateral optic neuritis. In 2011 he presented with low back pain, and urinary urgency, but his 
repeat MRI showed a smaller cervical cord lesion, extending from a smaller lesion, from $\mathrm{C} 2$ to $\mathrm{C6}_{5}$, and the previous thoracic lesion.

Two years following his last transverse myelitis TM, he underwent spinal cord MRS (Table 2); h h infor the right eye and blindness in the left-eye. He had moderately reduced power in his lower limbs, reduced sensory function in the left leg and urinary dysfunction.

\section{Case 5}

A 30-year old Chinese woman attended our clinic after moving to London. She had suffered seven episodes of TMservical myelitis over the previous eight years and had previously been diagnosed with MS.

Three months after her last transverse myelitis $\underline{\mathrm{TM}} \underline{\text { her spinal cord MRI showed a }}$ longitudinally-extensive lesion extending from the cranio-cervical junction to C7. her examination revealed a spastic tetraparesis, worse on the right, abnormal sensation below the neck and sensory ataxia. Spinal cord MRI showed a longitudinally-extensive lesion extending from the cranio-cervical junction to 67 . She tested positive for AQP4-Abs, and was re-diagnosed with NMO spectrum disorder. Her treatment was converted from interferon--beta 1-a to Azathioprine with Prednisolone. 
She then underwent spinal cord MRS. Examination revealed spastic tetraparesis, worse on the right, abnormal sensation below the neck and sensory ataxia.

\section{Differences in metabolite concentrations between groups}

Myo-Inositol/Creatine ratio was significantly lower in our case series of patients with NMO and NMO spectrum disorder than MS patients (Coeff. $-0.78, p<0.001$, 95\%Conf. Interval (Cl) $-1.24,-0.35$, obtained using the bootstrap) (Table

2)(Figure 1); additionally, patients with NMO showed significantly lower myoInositol/Creatine ratio than healthy controls (Coeff. $-0.45, p=0.008,95 \% \mathrm{Cl}-0.78$, 0.12) (Table 2)(Figure 1). Patients with MS showed higher No significant difference in-myo-Inositol/Creatine values than was found between MS patients and-healthy controls, but this did not reach statistical significance (Table 2)(Figure 1).

$\mathrm{N}$-acetyl-aspartate/Creatine values did not differ between NMO and MS, but were lower in MS than healthy controls (Coeff. $-0.48, \mathrm{p}<0.001,95 \% \mathrm{Cl}-0.76,-0.21$ )

(Table 2).

\section{Discussion}

This case series provides novel insights into the metabolic characteristics of LETM. Our key finding is that the levels of myo-Inositol (normalised to Creatine) of cervical cord lesions can discriminate between NMO/NMO spectrum disorders 
and MS. This may potentially have very important diagnostic implications. MyoInositol/Creatine values are reduced in the upper cervical cord NMO lesions when compared to healthy subjects (by about $28 \%$ ) and to MS patients with demyelinating lesions at the same cord level (by 40\%). Myo-Inositol is considered to be a marker of astrocytic activation and proliferation ${ }^{5}$; therefore, its reduction is likely to reflect astrocytic necrosis ${ }^{6}$, which is typically found in NMO lesions and is thought to mediate oligodrendrocyte injury ${ }^{7}$. Astrocytic damage per se, as reflected by elevated CSF glial fibrillary acidic protein, has been shown to be clinically relevant in $\mathrm{NMO}^{8}$.

The overall degree of tissue injury seen on conventional spinal cord MRI between $\underline{\mathrm{C} 1 \text { and } \mathrm{C} 3 \text { was overallless extensive in MS patients (see Supplemental Table }}$ 1) than NMO patients, but it is unlikely that this is the cause ofcontributed to the observed differenceschanges because healthy controls showed a MyoInositol/Creatine values which was-lower than in MS patients, but higher than in NMO patients. In particular,

Aalthough spinal cord MS lesions showed we detected a 17\% mean increase in Myo-Inositol/Creatine values than in spinal cord MS lesions when compared to healthy controls, this difference was not statistically significant; previous MRS studies reported increased myo-Inositol concentrations in the brain and spinal cord lesions in MS patients, when compared to healthy subjects ${ }^{9,10}$, reflecting the underlying process of gliosis. 
$\mathrm{N}$-acetyl-aspartate (NAA) is considered to be a marker of axonal integrity ${ }^{11}$ and/or metabolic function ${ }^{12}$, and is expected to be reduced in the presence of significant neuroaxonal degeneration and impaired metabolism. We found no significant differences in NAA/Creatine ratio between NMO patients and both healthy subjects and MS patients with at least one lesion at the same cord level. Conversely, MS patients showed a significantly lower mean NAA concentration than healthy controls (by about $39 \%$ ). These results are in agreement with previous studies, that reported (i) no significant spectroscopic abnormalities in the brain of NMO patients as compared to healthy controls ${ }^{13,14}$ and (ii) reduced levels of NAA in acute and chronic lesions in both the brain and spinal cord of MS patients when compared to healthy subjects ${ }^{4,15}$.

Our findings of abnormally low myo-Inositol levels in the LETM when compared to with MS patients and healthy subjects should encourage further investigation, since advanced spinal cord imaging could lead to novel imaging markers to distinguish NMO from MS and may have value in future clinical trials in NMO with remyelinating or neuroprotective agents.

\section{Acknowledgment}


This work was supported by the NIHR UCLH/UCL COMPREHENSIVE BIOMEDICAL RESEARCH CENTRE BRC. The authors thank Dr. Evelyne Balteau for her help with the shimming procedure. 


\section{References}

1. Wingerchuk DM, Lennon VA, Lucchinetti CF et al. The spectrum of neuromyelitis optica. Lancet Neurol. 2007; 6:805-815

2. Kinoshita $M$, Nakatsuji $Y$, Moriya $M$ et al. Astrocytic necrosis is induced by anti-aquaporin-4 antibody-positive serum. Neuroreport. 2009; 20:508-512

3. Hinson SR, Romero MF, Popescu BF et al. Molecular outcomes of neuromyelitis optica (NMO)-IgG binding to aquaporin-4 in astrocytes. Proc Natl Acad Sci U S A. 2012; 109:1245-1250

4. Ciccarelli O, Wheeler-Kingshott CA, McLean MA et al. Spinal cord spectroscopy and diffusion-based tractography to assess acute disability in multiple sclerosis. Brain. 2007; 130:2220-2231

5. Sajja BR, Wolinsky JS, Narayana PA. Proton magnetic resonance spectroscopy in multiple sclerosis. Neuroimaging Clin N Am. 2009; $19: 45-58$

6. Kinoshita $\mathrm{M}$, Nakatsuji $\mathrm{Y}$, Moriya $\mathrm{M}$ et al. Astrocytic necrosis is induced by anti-aquaporin-4 antibody-positive serum. Neuroreport. 2009; $20: 508-512$ 
7. Marignier R, Nicolle A, Watrin $\mathrm{C}$ et al. Oligodendrocytes are damaged by neuromyelitis optica immunoglobulin $\mathrm{G}$ via astrocyte injury. Brain. 2010; 133:2578-2591

8. Takano R, Misu T, Takahashi T et al. Astrocytic damage is far more severe than demyelination in NMO: A clinical CSF biomarker study. Neurology. 2010; 75:208-216

9. Marliani AF, Clementi V, Albini RL et al. Quantitative cervical spinal cord 3T proton MR spectroscopy in multiple sclerosis. AJNR Am J Neuroradiol. 2010; 31:180-184

10. Sajja BR, Wolinsky JS, Narayana PA. Proton magnetic resonance spectroscopy in multiple sclerosis. Neuroimaging Clin N Am. 2009; $19: 45-58$

11. Bjartmar C, Kidd G, Mork S et al. Neurological disability correlates with spinal cord axonal loss and reduced $\mathrm{N}$-acetyl aspartate in chronic multiple sclerosis patients. Ann Neurol. 2000; 48:893-901

12. Bates TE, Strangward M, Keelan J et al. Inhibition of $\mathrm{N}$-acetylaspartate production: implications for $1 \mathrm{H}$ MRS studies in vivo. Neuroreport. 1996; 7:1397-1400

13. De SJ, Blanc F, Kremer S et al. Magnetic resonance spectroscopy evaluation in patients with neuromyelitis optica. J Neurol Neurosurg Psychiatry. 2010; 81:409-411 
14. Pichiecchio A, Tavazzi E, Poloni G et al. Advanced magnetic resonance imaging of neuromyelitis optica: a multiparametric approach. Mult Scler. 2012; 18:817-824

15. Narayana PA, Doyle TJ, Lai D et al. Serial proton magnetic resonance spectroscopic imaging, contrast-enhanced magnetic resonance imaging, and quantitative lesion volumetry in multiple sclerosis. Ann Neurol. 1998; 43:56-71 
Table 1. Main clinical and radiological characteristics of NMO and NMO spectrum patients. TM=Transverse Myelitis, CSF=Corebrospinal Fluid, OCB=Oligoclonal Bands

\begin{tabular}{|c|c|c|c|c|c|c|c|}
\hline Patients & $\begin{array}{l}\text { Age } \\
\text { (yrs) } \\
\text { Gender }\end{array}$ & $\begin{array}{l}\text { Disease } \\
\text { duration }\end{array}$ & $\begin{array}{l}\text { AQP4- } \\
\text { Abs }\end{array}$ & $\begin{array}{l}\text { Time from last } \\
\underline{\text { TM }}\end{array}$ & $\begin{array}{l}\text { Conventional } \\
\text { Brain MRI }\end{array}$ & $\begin{array}{l}\text { Conventional } \\
\text { Spinal cord MRI }\end{array}$ & $\begin{array}{l}\text { CSF OCB } \\
\text { results }\end{array}$ \\
\hline $\begin{array}{l}\text { Case } 1 \\
\text { NMO } \\
\text { spectrum }\end{array}$ & $\begin{array}{l}38 \\
M\end{array}$ & 2 years & Positive & 17 months & Normal & $\begin{array}{l}\text { Lesion from C2 to } \\
\text { C5 and lesion in the } \\
\text { cranio-cervical } \\
\text { junction }\end{array}$ & Negative \\
\hline $\begin{array}{l}\text { Case } 2 \\
\text { NMO }\end{array}$ & $\begin{array}{l}44 \\
F\end{array}$ & 10 years & Positive & 12 months & $\begin{array}{l}\text { Normal } \\
\text { (except for } \\
\text { high T2 signal } \\
\text { in the right } \\
\text { optic nerve) }\end{array}$ & $\begin{array}{l}\text { Lesion from foramen } \\
\text { magnum to C6 }\end{array}$ & Negative \\
\hline $\begin{array}{l}\text { Case } 3 \\
\text { NMO } \\
\text { spectrum }\end{array}$ & $\begin{array}{l}50 \\
F\end{array}$ & $\begin{array}{l}4 \\
\text { months }\end{array}$ & Positive & 4 months & $\begin{array}{l}\text { Few, non- } \\
\text { enhancing, } \\
\text { white matter } \\
\text { lesions in the } \\
\text { periventricular } \\
\text { regions, pons }\end{array}$ & $\begin{array}{l}\text { Lesions at } \mathrm{C} 2 \text { and } \\
\mathrm{C} 3 / \mathrm{C} 4 \text {, and lesion } \\
\text { from } \mathrm{C} 6 \text { to the conus }\end{array}$ & $\begin{array}{l}\text { Positive in } \\
\text { the CSF, } \\
\text { negative in } \\
\text { the serum }\end{array}$ \\
\hline
\end{tabular}




\begin{tabular}{|c|c|c|c|c|c|c|c|}
\hline & & & & & $\begin{array}{l}\text { and corpus } \\
\text { callosum }\end{array}$ & & \\
\hline $\begin{array}{l}\text { Case } 4 \\
\text { NMO }\end{array}$ & $\begin{array}{l}34 \\
M\end{array}$ & 5 years & Positive & 24 months & $\begin{array}{l}\text { Non- } \\
\text { enhancing, } \\
\text { white matter } \\
\text { lesions in the } \\
\text { supratentorial } \\
\text { and } \\
\text { infratentotial } \\
\text { (pons and } \\
\text { medulla) } \\
\text { regions }\end{array}$ & $\begin{array}{l}\text { Lesion from } \mathrm{C} 2 \text { to } \\
\text { C6 and lesion from } \\
\text { T9 to T11 }\end{array}$ & Negative \\
\hline $\begin{array}{l}\text { Case } 5 \\
\text { NMO } \\
\text { spectrum }\end{array}$ & $\begin{array}{l}30 \\
F\end{array}$ & 8 years & Positive & 3 months & Normal & $\begin{array}{l}\text { Lesion from the } \\
\text { cranio-cervical } \\
\text { function to } \mathrm{C} 7\end{array}$ & Negative \\
\hline
\end{tabular}


Table 2. Metabolite concentrations in healthy subjects, patients with MS, and patients with either NMO or NMO spectrum disorder; results of the comparisons between groups.

\begin{tabular}{|l|l|l|l|l|l|l|}
\hline Ratios & Healthy & Patients & All & p-value & p-value & p-value \\
& subjects & with MS & patients & NMO & NMO & MS \\
with & vs. & vs. & vs. \\
& LETM & MS & controls & controls \\
NAA/Cr & $1.22(0.3)$ & $0.74(0.3)$ & $0.91(0.6)$ & n.s. & n.s. & $<0.001$ \\
$\begin{array}{l}\text { mean } \\
(S D)\end{array}$ & & $1.95(0.6)$ & $1.17(0.2)$ & 0.008 & $<0.001$ & n.s. \\
\hline $\begin{array}{l}\text { Ins/Cr } \\
\text { mean } \\
(S D)\end{array}$ & $1.62(0.5)$ & & & & & \\
\hline
\end{tabular}

Legend: * from Bootstrap analysis; $\mathrm{NAA}=\mathrm{N}$-acetyl-aspartate; $\mathrm{Cr}=$ Creatine plus Phosphocreatine; Ins= Myo-Inositol; n.s = non-significant. 
Figure 1. MR Spectroscopy of case 1 (NMO spectrum): (A) and (B) Spinal cord sagittal T2-weighted image showing a LETM between C2 and C5. (C) Location of the spectroscopic voxel at C1-C3 on the sagittal image. (D) Graph showing the mean values (and +/- 2SE) of Ins/Cr ratio in patients with NMO vs. patients with MS and healthy controls; (E) Patient's spectrum, showing reduced Ins/Cr (1.35) in comparison with a MS patient's spectrum $(\mathrm{Ins} / \mathrm{Cr}=1.69)(\mathbf{F})$ and healthy control's spectrum $(\mathrm{Ins} / \mathrm{Cr}=1.39)(\mathbf{G})$. 

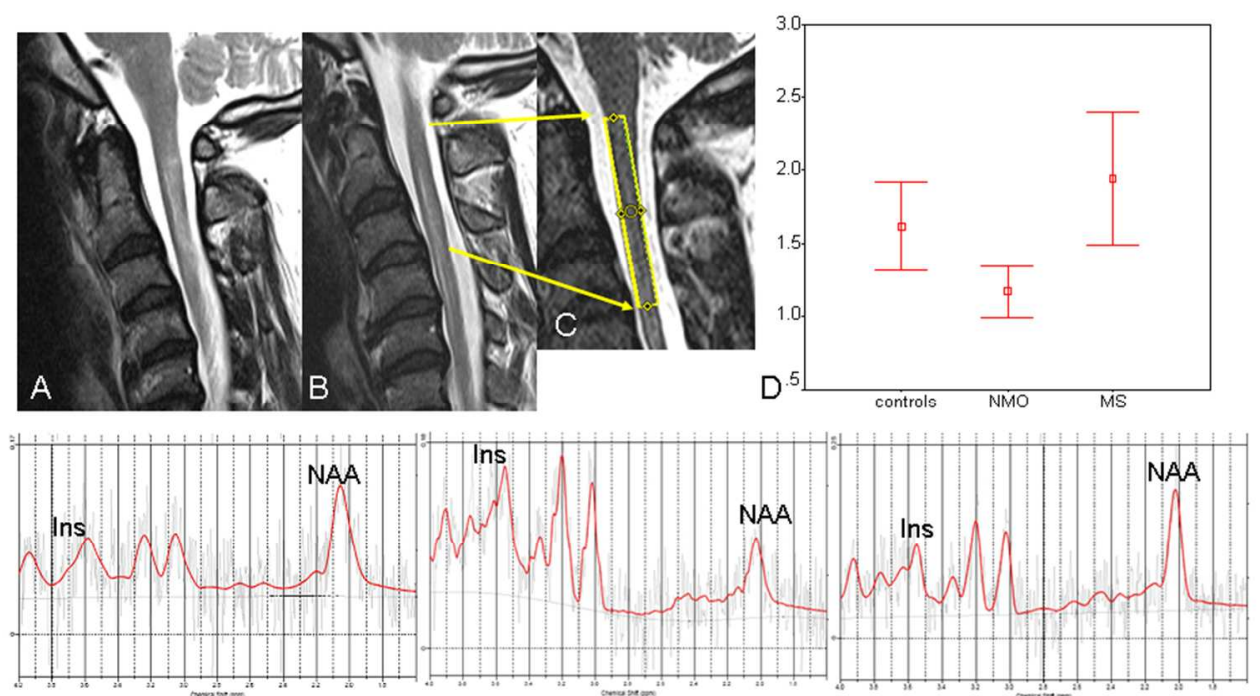

(E) Patient with NMO spectrum

(F) Patient with MS

(G) Healthy control

(A) and (B) Spinal cord sagittal T2-weighted image showing a LETM between C2 and C5.

(C) Location of the spectroscopic voxel at C1-C3 on the sagittal image. (D) Graph showing the mean values (and +/- 2SE) of Ins/Cr ratio in patients with NMO vs. patients with MS and healthy controls; (E) Patient's spectrum, showing reduced Ins/Cr (1.35) in comparison with a MS patient's spectrum (Ins/Cr=1.69) $(\mathrm{F})$ and healthy control's spectrum (Ins/ $\mathrm{Cr}=1.39)(\mathrm{G})$. $254 \times 190 \mathrm{~mm}(96 \times 96$ DPI) 


\section{Supplemental material}

\section{${ }^{1} \mathrm{H}$-MRS protocol and analysis}

Eleven healthy controls (age: 38.9yrs, SD 8.5), ten relapsing remitting MS

patients (age: 39yrs, SD 9.6) showing at least one demyelinating lesion involving the cervical cord region between C1 and C3 (Supplemental material - Table 1) and five patients with NMO or NMO spectrum disorder (age: 39.2yrs, SD 7.9) showing a LETM lesion involving either partially or completely the spine between $\underline{\mathrm{C} 1 \text { and } \mathrm{C} 3}$ underwent the same MRI protocol.

All MR data were collected on a Magnetom Tim Trio 3T system (Siemens AG, Erlangen, Germany), using the posterior half of a 12 channel receiver head coil, the posterior part of a neck array coil, and the upper element of the spine array coil.

After conventional T2 sagittal and coronal images, MRS was acquired with the PRESS sequence (TE=30ms; TR=3000ms; 160 averages), CHESS water suppression and cardiac gating. A non water-suppressed spectrum (2 averages) was also acquired for eddy-current correction. A single voxel was placed along the main axis of the cord between $\mathrm{C} 1$ and $\mathrm{C} 3$ on T2 -weighted sagittal images, as shown in Fig 1. Optimal shim currents were calculated off-line with in-house software. ${ }^{1}$ 
The analysis of the spectra was done using LCModel $^{2}$ using an analysis window between 1.6 and $4.0 \mathrm{ppm}$ and the ratios of the concentrations of the main metabolites, especially total $\mathrm{N}$-acetyl-aspartate (NAA)/ Creatine plus Phosphocreatine $[\mathrm{Cr}]$ and myo-Inositol $[\mathrm{Ins}] /[\mathrm{Cr}]$, were obtained. LCModel standard error estimates (\%SD, Cramer-Rao lower bounds) were used to assess the confidence of the concentration estimates. Spectra of three MS controls patients were excluded for low quality data (cases 8,9 and 10 in the Table). All the remaining spectra showed \%SD of [Cr] and [Ins] $<23 \%$ and \%SD of [NAA] $<25 \%$, except for case no. 2 (NMO) (SD of [NAA] 30\%) and case no. 4 (NMO) (SD of $[\mathrm{Ins}] 26 \%$ ). The average value of $[\mathrm{NAA}] /[\mathrm{Cr}]$ and $[\mathrm{Ins}] /[\mathrm{Cr}]$ in controls and in MS patients was calculated. The \% difference in these ratios and those obtained in the NMO and NMO spectrum disorder patients was calculated.

\section{Statistical analysis}

The differences in $[\mathrm{Ins}] /[\mathrm{Cr}]$ and $[\mathrm{NAA}] /[\mathrm{Cr}]$ between the three groups (i.e., NMO patients, MS patients and controls) were compared using the bootstrap, which is indicated when the sample is small and is distribution-independent. For each comparison, the observed coefficient, the $95 \%$ confidence interval and the $p$ value are reported.

References 
1. Balteau E, Hutton C, Weiskopf N., Improved shimming for fMRI specifically optimizing the local BOLD sensitivity. Neuroimage. 2010 Jan 1;49(1):327-36.

2. Provencher SW. Estimation of metabolite concentrations from localized in vivo proton NMR spectra. Magn Reson Med. 1993; 30:672-679 


\begin{tabular}{|c|c|c|}
\hline Patients & $\begin{array}{l}\text { Time from last } \\
\text { episode of } \\
\underline{\text { myelitis }}\end{array}$ & Conventional Spinal Cord MRI \\
\hline 1 & 10 months & $\begin{array}{l}\text { Demyelinating lesion at C1 and additional lesion at } \\
\underline{\mathrm{C} 2-\mathrm{C} 3 .}\end{array}$ \\
\hline$\underline{2}$ & 11 months & $\begin{array}{l}\text { Patchy lesions from C2-C3 down to the upper } \\
\text { thoracic cord. }\end{array}$ \\
\hline$\underline{3}$ & 3 months & $\begin{array}{l}\text { Multiple, patchy and short lesions in the cervical } \\
\text { cord involving C2-C3, C3-C4, C5 and C6-C7, T1, } \\
\text { T3 and T4-5. }\end{array}$ \\
\hline$\underline{4}$ & 4 months & $\begin{array}{l}\text { Discrete lesions in the cervical and thoracic cord (at } \\
\text { C2-C3, C7, T1-T3) and lower thoracic cord. }\end{array}$ \\
\hline$\underline{\mathbf{5}}$ & $\underline{60 \text { months }}$ & $\begin{array}{l}\text { Lesion at C2-C3 and further lesions at T3-T4 and } \\
\text { T11. }\end{array}$ \\
\hline$\underline{6}$ & 8 months & $\begin{array}{l}\text { Patchy lesions at C1, C2 and C4 and further lesions } \\
\text { at T6-T6 and T9. }\end{array}$ \\
\hline$\underline{7}$ & $\underline{6 \text { months }}$ & $\begin{array}{l}\text { Patchy lesions throughout the spinal cord, also } \\
\text { involving C2 and C3. }\end{array}$ \\
\hline$\underline{8}$ & 8 months & Lesion at $\mathrm{C} 2$. \\
\hline$\underline{9}$ & 24 months & Lesion at C2-C3 and C7-T3. \\
\hline$\underline{10}$ & 3 months & $\begin{array}{l}\text { Patchy lesions throughout the cord, in particular at } \\
\text { C1-C2, C3-C4 and C5-C5. }\end{array}$ \\
\hline
\end{tabular}


